\title{
Modeling spatiotemporal data: an issue in honor of Dr. Jean Paelinck
}

\author{
Jesús Mur • Fernando A. López
}

Received: 9 April 2010/Accepted: 14 April 2010/Published online: 8 May 2010

(C) Springer-Verlag 2010

Research in spatial econometrics has undergone a very intense increase in recent decades, both quantitatively and qualitatively. The indicators usually employed, such as published articles, handbooks and volumes edited and conferences and workshops organized, offer a robust image of this fast development. Another, and perhaps more important, aspect is the definite consolidation of this discipline as a fundamental tool for research in Economics. In this overall context, clearly optimistic, the problems associated with the specification of spatio-temporal models have attracted attention (Baltagi 2001). The scientific programs of recent international meetings, in regional economics and in spatial econometrics, with a growing increase in the number of sessions devoted to this topic is a good example for a tendency that, in our opinion, will continue in the near future. We need more techniques and more sophisticated tools to deal adequately with the larger and richer datasets that are currently available.

Our contribution to this discussion is modest but enthusiastic. We would like to mention the series of Jean Paelinck Seminars that began in October 2004, with the objective of honoring the mastery of Jean Paelinck in the fields of regional economics and spatial econometrics [let us mention the very well-known textbook by Paelinck and Klaassen (1979)]. The first and second seminars were held at the University of Zaragoza and the third one at the Technical University of Cartagena, between the 10th and the 12th of October, 2008. Twenty-five papers were presented there, some of which appear in the special issue of Journal of Geographical Systems briefly introduced here.

\footnotetext{
J. Mur

Departamento de Análisis Económico, Universidad de Zaragoza, Gran Vía 2-4, 50005 Zaragoza, Spain

F. A. López (ه)

Departamento Métodos Cuantitativos e Informáticos, Universidad Politécnica de Cartagena, C/Real, 3, 30201 Cartagena, Spain e-mail: fernando.lopez@upct.es
} 
This volume contains seven papers which have in common that they all deal with the modeling of spatio-temporal data. Three of these papers present methodological issues, whereas the other four present different applications to interesting problems. Four of them were first presented in the third seminar, and the other three respond to a challenge kindly accepted by the authors.

The contribution by Daniel Griffith, 'Modeling spatial temporal relationships: retrospect and prospect', is a good example of the main intention of this volume: to promote discussion on the state-of-the-art in the field of spatio-temporal modeling and to present some of the most promising future research lines. The situation that Griffith describes belongs to a field that is becoming very heterogeneous and can be divided into five areas of interest. These areas correspond to five methodological approaches: autoregressive-integrated moving average (ARIMA) models, space time autoregressive (STAR) models, geostatistical models, panel data models and spatial filter models. Clearly, the five approaches complement each other, and their utility and efficiency depend on the type of data available at each moment, and the purpose of a given data analysis (see Griffith 2003, for a thorough discussion). One of the more interesting facets of the filtering methodology consists in its flexibility, in its capacity to adapt to the use of strictly spatial data, strictly temporal data as well as to a pool of spatiotemporal data. As an example, this article reexamines the well-known case of sugarcane production in Puerto Rico. Griffith shows how the filtering technique yields results comparable with those from an ARIMA model in a time series setting. The extension to a panel data context is straightforward, simply combining the spatial and the temporal filters. The results, in terms of simplicity and explanatory power, seem quite promising. There remain a number of interesting points in the research agenda, as Griffith highlights. The development of a consistent distribution theory for space-time filters is among the most challenging. The obtaining of user-friendly visual interfaces for spatiotemporal data, the handling of missing space-time data values, a better incorporation of dynamics into panel data models, the use of non-normal probability models and the resolution of additional comparative analyses as well as additional case studies are other aspects that need more attention.

Valter di Giacinto, a well-known specialist in what has been termed spatial vector autoregressive (SpVAR) models, is the author of the paper 'On Vector Autoregressive modeling in Space and Time'. One of the main difficulties that a standard VAR user faces is the high number of parameters that appear in the model, the overparametrization problem (Sims 1980, is still an excellent reference). An excessive number of, sometimes, nuisance parameters hinder the identification of the structural parameters, the discussion of the causal relationships of interest and the efficient estimation of the whole model. A common solution consists in the introduction of a priori restrictions on the parameters to attain identification. The spatial framework to which the specification refers introduces an additional difficulty. The solution proposed by Di Giacinto is not without controversy. He advocates a recursive identification strategy using a block diagonal structure in the system of VAR equations. This means that the identification will depend on the introduction of different sets of restrictions, theoretical as well as spatial. This identification regime, popular in a time series context, assures the good behavior of 
the Full Information Maximum Likelihood estimates. As an additional result, once the SpVAR model is estimated, it will be possible to obtain the space-time impulse response functions. An application to a bivariate fiscal model for the case of the Italian regions illustrates the capability of SpVAR models.

Space is a domain that contains valuable information for modeling the relations between economic agents. This observation is a central point of consensus for practitioners of Spatial econometrics. But, to what extent? This is the point addressed by Angulo and Trívez in their paper 'The impact of spatial elements in the forecasting of Spanish labour series'. Specifically, the problem that they pose is the comparison, in terms of forecasting ability, of an absolutely aspatial technique against a second technique which heavily depends on the spatial structure of the data. The goal is to forecast the total employment for the 50 Spanish provinces in the year 2007. As the aspatial technique, the authors estimate fifty seasonal ARIMA models, one for each quarterly provincial series using the Box-Jenkins toolbox (Harvey 1990). In parallel, they also specify and estimate a single dynamic spatial panel data model using annual data. A dynamic spatial lag model with fixed effects appears to be the best option. One of the surprises of the exercise is that the spatial dynamic panel data model produces a forecast that is, at least, as accurate as the whole set of fifty ARIMA nonspatial models. This result confers a clear value to the explicit introduction of the spatial structure for modeling and forecasting spatial data.

The focus of the paper 'Public pharmaceutical expenditure: identification of spatial effects' is on the use of Seemingly Unrelated Regression equations introducing, once again, explicit spatial effects. The objective of the paper by Lauridsen, Bech and Maté is to explain the public pharmaceutical expenditures in the Spanish provinces in a somewhat short period, 1996-2003. They follow the approach of Anselin (1988, Chap. 10): one behavioral equation (pharmaceutical expenditures) specified under the assumption of homogeneity in the parameters, which is estimated using eight cross-sections over the fifty Spanish provinces. The authors allow for an unrestricted correlation structure between the error terms of the different cross-sections that result in the SUR mechanisms; they also allow for the existence of cross-sectional dependence among the provinces. The specification process leads the authors to a Spatially Distributed Lag model with an autoregressive term in the right-hand side of the equation and a SUR mechanism in the error term. This specification confirms the existence of spatial spill-over effects in the pharmaceutical expenditures combined with a strong temporal inertia.

Beenstock and Felsenstein present the paper 'Spatial error correction and cointegration in nonstationary panel data: regional house prices in Israel'. The authors provide a comprehensive introduction to this kind of models, including a taxonomy of spatial error correction models, SpECM. They distinguish between local, spatial and global cointegration relations in a spatial model that combines with another three types of error correction mechanism, of a local, spatial or global nature. In an unrestricted framework, there are nine possibilities.

Beenstock and Felsenstein discuss how to 'spatialize' error correction mechanisms estimated from general nonstationary panel data models according to an innovative research proposal that combines cross-sectional data with time series models (in line with Pesaran and Smith 1995). The paper contains a very interesting 
application to the case of house prices in Israel. Specifically, the authors test whether the spatial lag of regional house prices belongs to the cointegrated vector as predicted by the theory. The results strongly support this view as well as the assumption of global cointegration in the Israeli regional house market.

Márquez, Ramajo and Hewings develop a novel methodological procedure to assess the impact of production externalities across regional economies by means of a dynamic spatio-temporal panel error correction model in 'A spatio-temporal econometric model of regional growth in Spain'. The procedure works well with a small number of spatial entities for which there are a large number of observations in the time dimension (Phillips and Moon 2000). The authors apply this procedure to the case of the Spanish Autonomous Communities, obtaining a cointegrating relationship between the level of regional output and the level of regional factors. Then, they estimate a dynamic spatio-temporal panel error correction model to describe the shortrun regional growth adjustment process in space and in time. This approach seems to offer a clear advantage over standard regional growth models, as shown in the paper. The result is very important for applied studies where it is frequent to find cases in which the time dimension is large, whereas the spatial cross-section is reduced.

In spite of it formal elegancy, the Bayesian approach occupies a minority position in the spatial econometrics toolbox (Lesage and Pace 2009); this is also true for the case of spatio-temporal analysis. The paper by Beamonte, Gargallo and Salvador, 'Analysis of housing price by means of STAR models with neighbourhood effects: A Bayesian approach', is a good example of the capacity of this type of technique. The application presented by the authors to the real-estate market of an urban environment, the city of Zaragoza, shows that it is possible to make precise inferences about the parameters, taking into account the uncertainty associated with the estimation algorithms; the goal is to avoid the use of asymptotic results of doubtful validity in most of the cases. This paper is an extension of previous research by the same authors. They introduce larger and denser nets, in order to capture more adequately the influence of spatial and temporal neighbors, and a continuous treatment of the smoothing weights which increases the flexibility and generality of the method.

Finally, we would like to thank everyone who has taken part in the seminar in any of its editions. Thanks also to the authors and referees who have intervened in the editing of this special issue which would not have been possible without the continuous and devoted interest of many others, in particular to the Editors-in-Chief of Journal of Geographical Systems, Manfred M. Fischer and Antonio Páez, and the Managing Editor, Thomas Seyffertitz. The financial support of projects SEJ200602328, ECO2009-10534 and SEJ2007-30277-E/ECON is also recognized as well as the scientific support of the Spatial Econometrics Association and the Spanish Regional Science Association.

\section{References}

Anselin L (1988) Spatial econometrics: methods and models. Kluwer, Dordrecht Baltagi B (2001) Econometric analysis of panel data. Wiley, New York 
Griffith D (2003) Spatial autocorrelation and spatial filtering. Gaining understanding through theory and scientific visualization. Springer, Berlin

Harvey A (1990) The econometric analysis of time series. Philip Allan, Hempstead

LeSage J, Pace K (2009) Introduction to spatial econometrics. CRC Press, Boca Ratón

Paelinck J, Klaassen L (1979) Spatial econometrics. Saxon House, Farnborough

Pesaran H, Smith R (1995) Estimating long-run relationships from dynamic heterogeneous panels. J Econom 68(1):79-113

Phillips P, Moon H (2000) Nonstationary panel data analysis: an overview of some recent developments. Econom Rev 19(3):263-286

Sims C (1980) Macroeconomics and reality. Econometrica 48(1):1-48 